\title{
What Is Injury in Ice Hockey: An Integrative Literature Review on Injury Rates, Injury Definition, and Athlete Exposure in Men's Elite Ice Hockey
}

\author{
Anthony S. Donskov ${ }^{1,2, *(\mathbb{D})}$, David Humphreys ${ }^{1}$ and James P. Dickey ${ }^{1}$ (D) \\ 1 Department of Kinesiology, University of Western Ontario, London, ON N6A 357, Canada; \\ dhumphr4@uwo.ca (D.H.); jdickey@uwo.ca (J.P.D.) \\ 2 Donskov Strength \& Conditioning, Columbus, OH 43229, USA \\ * Correspondence: adonskov@uwo.ca
}

Received: 18 September 2019; Accepted: 18 October 2019; Published: 23 October 2019

check for updates

\begin{abstract}
Injuries in men's elite ice hockey have been studied over the past 40 years, however, there is a lack of consensus on definitions of both injury and athlete exposure. These inconsistencies compromise the reliability and comparability of the research. While many individual studies report injury rates in ice hockey, we are not aware of any literature reviews that have evaluated the definitions of injury and athlete exposure in men's elite ice hockey. The purpose of this integrative review was to investigate the literature on hockey musculoskeletal injury to determine injury rates and synthesize information about the definitions of injury and athlete exposure. Injury rates varied from 13.8/1000 game athlete exposures to 121/1000 athlete exposures as measured by player-game hours. The majority of variability between studies is explained by differences in the definitions of both injury and athlete exposure. We were unable to find a consensus injury definition in elite ice hockey. In addition, we were unable to observe a consistent athlete exposure metric. We recommend that a consistent injury definition be adopted to evaluate injury risk in elite ice hockey. We recommend that injuries should be defined by a strict list that includes facial lacerations, dental injuries, and fractures. We also recommend that athlete exposure should be quantified using player-game hours.
\end{abstract}

Keywords: sports injuries; injury surveillance; lower extremity injuries; professional hockey players

\section{Introduction}

Ice hockey is a high intensity sport where players can reach speeds of up to $48 \mathrm{kph}$ [1]. These speeds, and the nature of collision sports lead to musculoskeletal injuries at all levels of ice hockey [1-3]. There is a need to accurately quantify injury rates in men's elite ice hockey both for assessing player risk [4] and the associated economic burden [5]. Injury rates in ice hockey have been investigated in order to assess injury trends, injury types, injury location, and underlying injury mechanisms [6]. Injury rates can also be used to quantify the effects of rule changes [7]. Accurate data is needed in order to better investigate areas of concern while objectifying the effects of rule changes and other preventative measures $[8,9]$.

Differences in the definitions for injury and athlete exposure (AE) lead to inconsistencies between studies, and obscure the resulting injury rates. Consensus statements on injury definitions and data collection procedures have been developed for soccer [10] and rugby [11], but have not been developed for ice hockey. Consistent definitions and methods to evaluate ice hockey injuries are required [12] to improve the comparability of published data [8]. Our objective was to review global musculoskeletal injury rates in men's elite ice hockey, as well as definitions of injury and athlete exposure. We focused our review on males as females have different types and rates of injury than 
males [13]. We focused on elite players aged 16 years and older playing junior hockey (United States Hockey League, North American Hockey League, Canadian Hockey League), US and Canadian College Hockey (NCAA Div. 1 and Div. III, Canadian Inter-University Sport), international or minor professional and professional hockey (Finnish Elite League, Swedish Elite League, Japanese Elite League, International Ice Hockey and the National Hockey League) as this cohort has not been as extensively studied as other levels such as high school and youth hockey $[14,15]$. In addition, the economic burden of injury at this level is high. During two seasons in the National Hockey League (NHL), injuries represented a total salary cost of US $\$ 218$ million per year. While salary losses represent a significant financial burden, it is hoped that improved injury surveillance will reduce these costs.

\section{Materials and Methods}

We conducted an integrative literature review [16] to evaluate musculoskeletal injury rates, injury definition and athlete exposure measurement in elite ice hockey. We formulated three research questions a priori to focus our review: What is the rate of musculoskeletal injuries in men's elite ice hockey? In elite ice hockey, what injury definition is best suited to enable direct comparisons among research studies? Finally, in elite ice hockey, what measure of athlete exposure is best suited to achieve consistent and comparable injury rates?

\section{Literature Search}

A PubMed search strategy was created with the assistance of a University research librarian. PubMed was chosen as a search engine as it is the optimal tool in life sciences and biomedicine [17]. The search strategy used the key words: hockey AND (injury OR injuries) AND (NHL OR national OR international OR world OR competitive OR professional OR elite OR high caliber OR high caliber OR collegiate OR university OR intercollegiate OR NCAA OR "National Collegiate Athletic Association"). In addition, the same search strategy was performed on SPORTDiscus. The PubMed and SPORTDiscus records of these references were pooled and screened based on established inclusion and exclusion criteria (Table 1). Articles that were not relevant to our research questions were excluded. The references in the remaining papers were reviewed to identify additional relevant articles. All studies were reviewed by both authors for their relevance to the three research questions.

Original, peer-reviewed, English language research articles evaluating the injury rates in elite ice hockey were included. Articles were excluded if they were editorials, abstracts, books or excerpts from conference proceedings. Articles were excluded if they did not contain one of the following variables: injury definition, injury rate, athlete exposure, injury mechanism or injury location. Unpublished data was not reviewed. 
Table 1. Inclusion/exclusion criteria for literature search

\begin{tabular}{|c|c|c|c|}
\hline Factor & Inclusion Criteria & Exclusion Criteria & Rationale for This Criterion \\
\hline Publication Type & $\begin{array}{l}\text { Peer-reviewed original research articles } \\
\text { only }\end{array}$ & $\begin{array}{l}\text { Review papers, non-peer reviewed } \\
\text { articles, editorials, abstracts, book } \\
\text { chapters and conference proceedings }\end{array}$ & $\begin{array}{l}\text { For practical reasons, it was deemed to exclusively review } \\
\text { primary research articles, rather than non-peer reviewed or } \\
\text { abbreviated sources. }\end{array}$ \\
\hline Language & English language & Non-English & $\begin{array}{l}\text { For practical reasons, it was deemed acceptable to only } \\
\text { include studies published in English. }\end{array}$ \\
\hline Publication Date & November 1976 to April 2019 & Publications prior to January 1975 & $\begin{array}{l}\text { The characteristics of ice hockey injury reporting may } \\
\text { change over time due to rule changes, technological } \\
\text { advancements and education. Literature was captured } \\
\text { backdated to } 1988 \text { to capture these potential developments. }\end{array}$ \\
\hline Study Design & $\begin{array}{l}\text { Multi-center studies, randomized control } \\
\text { trials, cohort studies, case-controlled } \\
\text { studies and cross-sectional studies. }\end{array}$ & Case studies & $\begin{array}{l}\text { Study design was chosen to ensure reasonable empirical } \\
\text { support, and high methodological rigor in defining injury } \\
\text { and injury rates amongst competitive hockey players. }\end{array}$ \\
\hline Gender and Age & $\begin{array}{l}\text { Men athletes aged }>16 \text {-years } \\
\text { participating in a competitive } \\
\text { league/team }\end{array}$ & $\begin{array}{l}\text { Women only studies or men ages }<16 \text {, } \\
\text { age unspecified involved in youth sport }\end{array}$ & $\begin{array}{l}\text { The primary outcome of interest was injury definition and } \\
\text { injury rate calculation in competitive ice hockey played by } \\
\text { men. Studies that compared rates between males and } \\
\text { females and have separate data for both genders were also } \\
\text { included for baseline comparisons. } \\
\text { Men athletes aged }>16 \text { were considered appropriate. This } \\
\text { age demographic represents elite players. }\end{array}$ \\
\hline Playing Level & Competitive participation & Recreational sport/training & $\begin{array}{l}\text { The primary outcomes of interest are injury definition, } \\
\text { injury rates, mechanism and anatomical location sustained } \\
\text { during competitive ice hockey. }\end{array}$ \\
\hline Sport & $\begin{array}{l}\text { Injuries must be sustained during ice } \\
\text { hockey games and practices }\end{array}$ & Any sport other than ice hockey & $\begin{array}{l}\text { Sports included other than ice hockey may result in } \\
\text { definitions, and injury rates that are too broad. }\end{array}$ \\
\hline Types of Injury & $\begin{array}{l}\text { Injuries to the musculoskeletal system, } \\
\text { including strains, sprains, breaks }\end{array}$ & $\begin{array}{l}\text { Concussions, spinal injuries, head/face, } \\
\text { lacerations }\end{array}$ & $\begin{array}{l}\text { The primary outcomes of interest are soft tissue injuries of } \\
\text { the upper and lower extremity during competitive ice } \\
\text { hockey }\end{array}$ \\
\hline Outcome Measures & $\begin{array}{l}\text { Injury definition, injury rates, athlete } \\
\text { exposure, mechanisms, anatomical } \\
\text { location }\end{array}$ & $\begin{array}{l}\text { Outcomes other than injury definition, } \\
\text { injury rate, and athlete exposure, } \\
\text { mechanisms and anatomical location }\end{array}$ & $\begin{array}{l}\text { The primary outcomes of interest are injury definition, } \\
\text { injury rates, mechanisms and anatomical location. }\end{array}$ \\
\hline
\end{tabular}

All studies were reviewed by both authors. The study methodology, and outcome measures were extracted. 


\section{Results}

The PubMed and SPORTDiscus search identified 2463 references. An additional 3 pertinent articles were identified from the references for these articles. A total of 2212 articles were vetted after 254 duplicate articles were removed. Two-thousand, one-hundred and eighty-four of these articles were excluded as they were not relevant to any of our three research questions. No relevant articles were published prior to 1975. Accordingly, a total of 28 articles were included (Figure 1).

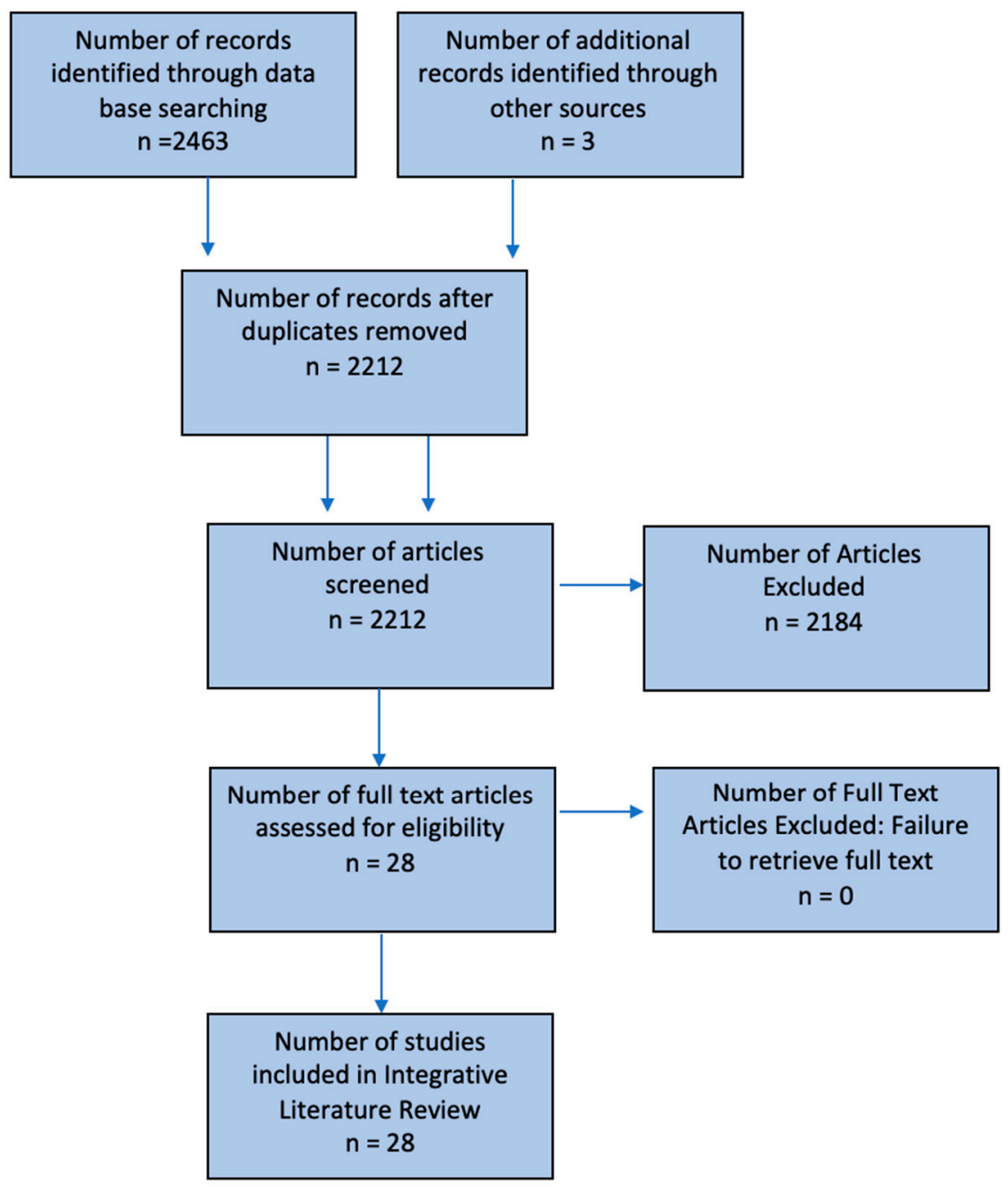

Figure 1. Flowchart describing the process for selecting relevant studies. The top row represents the identification process. The second and third rows represent the screening process. The fourth row represents the eligibility of the articles assessed and the last row identifies the articles included.

\subsection{Rate of Musculoskeletal Injuries in Men's Elite Ice Hockey (Question \#1)}

Injury rate data, and study design characteristics are presented for each of the 28 studies in Table 2. Injury rates in competitive ice hockey range from 13.8 to 121/1000 player-game hours, depending on factors such as the league of play and exposure estimate. Professional players in Europe and North America experience musculoskeletal injury rates between 49 to $80 / 1000 \mathrm{AE}$ as measured in player-game hours $[4,18]$ while the collegiate hockey players in Canada and the United States experience lower rates (13.8 to 19.95/1000 AE) as measured in player games [19,20]. The highest injury rates are experienced at the junior level (39.8 to 121/1000 player-game hours) [21,22]. The majority of these musculoskeletal injuries are attributed to collision with other players, the boards or the hockey puck $[18,20,23,24]$. 
Table 2. Summary of papers evaluating injury definition, injury rate, athlete exposure and injury mechanism in men's elite ice hockey.

\begin{tabular}{|c|c|c|c|c|c|c|c|c|}
\hline Authors & Year & Demographic & Injury Definition & Type & Injury Rate & $\begin{array}{c}\text { Mechanism of } \\
\text { Injury }\end{array}$ & Injury Type & $\begin{array}{c}\text { Injury Rate } \\
\text { Computation }\end{array}$ \\
\hline Hayes [25] & 1975 & $\begin{array}{l}\text { Intercollegiate } \\
\text { Ice hockey }\end{array}$ & $\begin{array}{l}\text { "An event requiring } \\
\text { some attention by the } \\
\text { team trainer or physician } \\
\text { or both." }\end{array}$ & $\begin{array}{l}\text { Medical } \\
\text { Attention }\end{array}$ & $\begin{array}{l}1.14 \text { injuries per } \\
\text { game (Canada) } \\
1.28 \text { injuries per } \\
\text { game (USA) }\end{array}$ & Collision & $\begin{array}{l}\text { Head and face, knee, } \\
\text { shoulders }\end{array}$ & $\begin{array}{l}\text { Total } \\
\text { injuries/Total } \\
\text { number of games }\end{array}$ \\
\hline $\begin{array}{l}\text { Sutherland } \\
\text { [26] }\end{array}$ & 1976 & Youth-Pro & $\begin{array}{l}\text { The injuries were } \\
\text { classified according to the } \\
\text { standard nomenclature of } \\
\text { athletic injuries as } \\
\text { recommended by the } \\
\text { American Medical } \\
\text { Association }\end{array}$ & $\mathrm{N} / \mathrm{A}$ & $\begin{array}{l}\text { Pro Group: } \\
\text { 143/1000 AE } \\
\text { (practice and } \\
\text { games) }\end{array}$ & $\mathrm{N} / \mathrm{A}$ & $\begin{array}{l}\text { Scalp and face } 60.8 \% \text {, } \\
\text { Groin } 9.1 \% \text {, } \\
\text { knee } 7.8 \% \text {, } \\
\text { shoulder } 5.9 \%\end{array}$ & $\mathrm{~N} / \mathrm{A}$ \\
\hline $\begin{array}{l}\text { Hayes } \\
{[27]}\end{array}$ & 1978 & Youth-Pro & $\begin{array}{l}\text { "Any change in the } \\
\text { normal, healthy state of } \\
\text { the individual that } \\
\text { requires medical } \\
\text { attention and disables a } \\
\text { player either temporarily } \\
\text { or permanently." }\end{array}$ & $\begin{array}{l}\text { Medical } \\
\text { Attention }\end{array}$ & $\begin{array}{l}\text { University: } \\
\text { 1.17/Game } \\
\text { Professional: } \\
\text { 1.15/Game }\end{array}$ & $\begin{array}{l}\text { Stick and puck } \\
\text { contact }\end{array}$ & $\begin{array}{l}\text { Contusions and } \\
\text { lacerations }\end{array}$ & $\begin{array}{l}\text { Total } \\
\text { injuries/Total } \\
\text { number of games }\end{array}$ \\
\hline $\begin{array}{l}\text { Rielly } \\
{[28]}\end{array}$ & 1982 & College Hockey & $\begin{array}{l}\text { "A reportable injury was } \\
\text { defined as being one that } \\
\text { required definitive } \\
\text { physical evaluation and } \\
\text { medical treatment." }\end{array}$ & $\begin{array}{l}\text { Medical } \\
\text { Attention } \\
\text { Definition }\end{array}$ & $1 / 12.7 \mathrm{~h}$ of play ${ }^{* *}$ & $\begin{array}{l}\text { Player contact } \\
(43.3 \%), \text { puck } \\
\text { contact } 27 \%\end{array}$ & Face, hips, shoulders & N/A \\
\hline $\begin{array}{l}\text { Meeuwisse } \\
\text { et al. } \\
\text { [29] }\end{array}$ & 1988 & $\begin{array}{l}\text { Canadian } \\
\text { University }\end{array}$ & $\begin{array}{l}\text { Injury was defined as any } \\
\text { disability arising either in } \\
\text { practice or competition } \\
\text { that required physical } \\
\text { attention. }\end{array}$ & $\begin{array}{l}\text { Medical } \\
\text { Attention } \\
\text { Definition }\end{array}$ & $\begin{array}{l}\text { As calculated by } \\
\text { percentage. } \\
\text { Hockey had the } \\
\text { greatest } \\
\text { percentage of } \\
\text { players injured. }\end{array}$ & $\mathrm{N} / \mathrm{A}$ & Knee, ribs, low back & $\mathrm{N} / \mathrm{A}$ \\
\hline
\end{tabular}


Table 2. Cont

\begin{tabular}{|c|c|c|c|c|c|c|c|c|}
\hline Authors & Year & Demographic & Injury Definition & Type & Injury Rate & $\begin{array}{l}\text { Mechanism of } \\
\text { Injury }\end{array}$ & Injury Type & $\begin{array}{c}\text { Injury Rate } \\
\text { Computation }\end{array}$ \\
\hline $\begin{array}{l}\text { Lorenzton, } \\
\text { Wedren, } \\
\text { Pietila [30] }\end{array}$ & 1988 & $\begin{array}{l}\text { Swedish Elite } \\
\text { Team }\end{array}$ & $\begin{array}{l}\text { "Injury was defined as } \\
\text { any injury occurring } \\
\text { during on-ice practice or } \\
\text { games and causing the } \\
\text { player to miss the next } \\
\text { practice session or game. } \\
\text { Facial lacerations, which } \\
\text { are common in ice hockey } \\
\text { but do not cause absence } \\
\text { from practice or game are } \\
\text { reported separately." }\end{array}$ & $\begin{array}{l}\text { Time Loss } \\
\text { Definition }\end{array}$ & $\begin{array}{l}78.4 / 1000 \text { player } \\
\text { game hours, } \\
1.4 / 1000 \text { player } \\
\text { practice hours }\end{array}$ & $\begin{array}{l}\text { Checking } 32.9 \% \text {, } \\
\text { Player contact } 25 \% \text {, } \\
\text { Puck contact } 14.5 \% \text {, } \\
\text { stick contact } 11.8 \% \text {, } \\
\text { collision with } \\
\text { boards } 6.6 \% \text {, } \\
\text { cutting, } 6.6 \% \text {, } \\
\text { skate contact } 2.6 \%\end{array}$ & $\begin{array}{l}\text { Contusions, strains and } \\
\text { sprains were the most } \\
\text { common types of injury. } \\
\text { Knees were the most } \\
\text { commonly injured joint } \\
\text { (5 injuries were } \\
\text { complete tears of the } \\
\text { MCL). } 53.7 \% \text { of injuries } \\
\text { were localized in the } \\
\text { lower limb. }\end{array}$ & $\begin{array}{l}\text { Practice Injury } \\
\text { Rate = number of } \\
\text { injuries/Practice } \\
\text { Hours } \times \text { Roster } \\
(23.4) . \\
\text { Games Injury } \\
\text { Rate }=\text { number of } \\
\text { injuries/Game } \\
\text { hours } \times \text { Total } \\
\text { Players on ice (6). }\end{array}$ \\
\hline $\begin{array}{l}\text { Lorenzton, } \\
\text { Wedren, } \\
\text { Pietila, } \\
\text { Gustavsson } \\
\text { [18] }\end{array}$ & 1988 & $\begin{array}{l}\text { Swedish } \\
\text { National Team } \\
\text { (40 International } \\
\text { games) }\end{array}$ & $\begin{array}{l}\text { "Injury was defined as } \\
\text { any injury occurring } \\
\text { during games and } \\
\text { causing the player to } \\
\text { miss the next practice } \\
\text { session or game. Facial } \\
\text { lacerations, which are } \\
\text { common in ice hockey } \\
\text { but do not cause absence } \\
\text { from practice or game are } \\
\text { reported separately." }\end{array}$ & $\begin{array}{l}\text { Time Loss } \\
\text { Definition }\end{array}$ & $\begin{array}{l}\text { 79.2/1000 player } \\
\text { game hours }\end{array}$ & $\begin{array}{l}\text { Player contact } \\
42.1 \% \text {, } \\
\text { checking } 31.6 \% \text {, } \\
\text { collision with } \\
\text { boards } 10.5 \% \text {, } \\
\text { puck/skate contact } \\
5.3 \%\end{array}$ & $\begin{array}{l}\text { Contusions, strains and } \\
\text { sprains were the most } \\
\text { common types of injury. } \\
\text { Knees were the most } \\
\text { commonly injured joint, } \\
\text { followed by the thigh } \\
\text { and wrist. }\end{array}$ & $\begin{array}{l}\text { Total } \\
\text { Injuries/Total AE } \\
\times 1000 ; \\
\text { Total AE = games } \\
\times \text { Total players on } \\
\text { ice (6) }\end{array}$ \\
\hline $\begin{array}{l}\text { Tegner, } \\
\text { Lorentzon } \\
{[24]}\end{array}$ & 1991 & $\begin{array}{l}\text { Swedish Elite } \\
\text { League (12 } \\
\text { teams) }\end{array}$ & $\begin{array}{l}\text { "Injury was defined as } \\
\text { any injury occurring } \\
\text { during ice practices or } \\
\text { games and causing the } \\
\text { player to stop playing or } \\
\text { to miss the next practice } \\
\text { session or game. Facial } \\
\text { lacerations, which are } \\
\text { common in ice hockey, } \\
\text { but do not cause absence } \\
\text { from practice or games, } \\
\text { are also reported." }\end{array}$ & $\begin{array}{l}\text { Time Loss } \\
\text { Definition }\end{array}$ & $\begin{array}{l}53 / 1000 \text { player } \\
\text { game hours }(76 \% \\
\text { of injuries } \\
\text { occurred during } \\
\text { games) }\end{array}$ & $\begin{array}{l}\text { Stick contact } 25.5 \% \text {, } \\
\text { player contact } 24 \% \text {, } \\
\text { puck contact } 11.2 \% \text {, } \\
\text { collision with } \\
\text { boards or goal posts } \\
9.7 \%\end{array}$ & $\begin{array}{l}\text { Strain, laceration and } \\
\text { contusions were the } \\
\text { most common types of } \\
\text { injury. Knees were the } \\
\text { most common joint } \\
\text { injured }(13.2 \%) \text {, } \\
\text { followed by the hip } \\
(12.1 \%)\end{array}$ & $\begin{array}{l}\text { Total } \\
\text { Injuries/Total AE } \\
\times 1000 \\
\text { Total AE = games } \\
\times \text { Total players on } \\
\text { ice (6) }\end{array}$ \\
\hline
\end{tabular}


Table 2. Cont.

\begin{tabular}{|c|c|c|c|c|c|c|c|c|}
\hline Authors & Year & Demographic & Injury Definition & Type & Injury Rate & $\begin{array}{l}\text { Mechanism of } \\
\text { Injury }\end{array}$ & Injury Type & $\begin{array}{l}\text { Injury Rate } \\
\text { Computation }\end{array}$ \\
\hline $\begin{array}{l}\text { McKnight, } \\
\text { Ferrara, } \\
\text { Czerwinska } \\
\text { [23] }\end{array}$ & 1992 & $\begin{array}{l}\text { Collegiate (Div. } \\
\text { I) }\end{array}$ & $\begin{array}{l}\text { (1). Loss of practice or } \\
\text { game time because of } \\
\text { injury/illness, (2). Injury } \\
\text { that required sutures } \\
\text { even if no time loss was } \\
\text { involved, (3). Injury in } \\
\text { which a fracture or } \\
\text { dislocation/subluxation } \\
\text { occurred even if the } \\
\text { athlete was able to } \\
\text { continue participation }\end{array}$ & $\begin{array}{l}\text { Time } \\
\text { Loss/Medical } \\
\text { Attention } \\
\text { Definition }\end{array}$ & $\begin{array}{l}\text { Total: } 10.22 / 1000 \\
\text { AE Games: } \\
14.73 / 1000 \text { game } \\
\text { hours. Practice: } \\
2.52 / 1000 \text { practice } \\
\text { hours }\end{array}$ & $\begin{array}{l}\text { Person/Ice Impact } \\
42 \% \text {, impact with } \\
\text { the boards } 32 \% \text {. The } \\
\text { shoulder and knee } \\
\text { had the highest rate } \\
\text { of injury when } \\
\text { compared to other } \\
\text { body parts }\end{array}$ & $\begin{array}{l}\text { Contusions and strains } \\
\text { were the most common } \\
\text { types of injury }\end{array}$ & $\begin{array}{l}\text { number of } \\
\text { Injuries/Total AE } \\
\times 1000 \text { (games } \\
\text { and practice) }\end{array}$ \\
\hline $\begin{array}{l}\text { Pelletier, } \\
\text { Montelpare, } \\
\text { Stark [20] }\end{array}$ & 1993 & $\begin{array}{l}\text { Canadian } \\
\text { Inter-collegiate }\end{array}$ & $\begin{array}{l}\text { "Any brain concussion } \\
\text { causing cessation of the } \\
\text { athlete's participation for } \\
\text { physical observation } \\
\text { before return to play, any } \\
\text { dental injury requiring } \\
\text { professional attention, } \\
\text { any injury/illness causing } \\
\text { cessation of an athlete's } \\
\text { customary participation } \\
\text { throughout the } \\
\text { participation day } \\
\text { following day of onset, or } \\
\text { any injury/illness } \\
\text { requiring substantive } \\
\text { professional attention } \\
\text { before the athlete's return } \\
\text { to competition." }\end{array}$ & $\begin{array}{l}\text { Time } \\
\text { Loss/Medical } \\
\text { Attention } \\
\text { Definition }\end{array}$ & $\begin{array}{l}\text { 19.95/1000 AE } \\
\text { (player games) }\end{array}$ & $\begin{array}{l}\text { Body checking } \\
44.6 \% \text {, collision } \\
\text { (accidental) } 28.8 \% \text {, } \\
\text { stick } 12.2 \% \text {, fighting } \\
6.5 \% \text {, illegal body } \\
\text { check } 5.8 \% \text {, } \\
\text { non-contact } 2.2 \%\end{array}$ & $\begin{array}{l}\text { Sprains }(31 \%) \text { and } \\
\text { contusions }(21 \%) \text { were } \\
\text { the most common type } \\
\text { of injury. } \\
\text { Knees were most } \\
\text { frequently injured } \\
(18.6 \%) \text {, } \\
\text { followed by teeth and } \\
\text { eyes }(17.6 \%), \\
\text { and shoulders }(14.9 \%),\end{array}$ & $\begin{array}{l}\text { Total } \\
\text { Injuries/Total AE } \\
\times 1000 \\
\text { Total AE = games } \\
\times \text { Total players on } \\
\text { team }(19)\end{array}$ \\
\hline
\end{tabular}


Table 2. Cont

\begin{tabular}{|c|c|c|c|c|c|c|c|c|}
\hline Authors & Year & Demographic & Injury Definition & Type & Injury Rate & $\begin{array}{l}\text { Mechanism of } \\
\text { Injury }\end{array}$ & Injury Type & $\begin{array}{l}\text { Injury Rate } \\
\text { Computation }\end{array}$ \\
\hline $\begin{array}{l}\text { Pettersson, } \\
\text { Lorentzon } \\
{[31]}\end{array}$ & 1993 & $\begin{array}{l}\text { Swedish Elite } \\
\text { League }\end{array}$ & $\begin{array}{l}\text { "Injury was defined as } \\
\text { any injury occurring } \\
\text { during on-ice practice or } \\
\text { games and requiring } \\
\text { medical attention and } \\
\text { treatment. Injuries } \\
\text { causing the player to } \\
\text { miss the next practice or } \\
\text { game have been analyzed } \\
\text { separately." }\end{array}$ & $\begin{array}{l}\text { Medical } \\
\text { Attention } \\
\text { Definition }\end{array}$ & $\begin{array}{l}74.1 / 1000 \text { game } \\
\text { hours }\end{array}$ & $\begin{array}{l}\text { Stick contact } 26.1 \% \text {, } \\
\text { player contact } \\
23.9 \% \text {, } \\
\text { puck contact } 16 \% \text {, } \\
\text { collision with } \\
\text { boards } 7.2 \% \text {, } \\
\text { fall (no contact) } 4 \%\end{array}$ & $\begin{array}{l}\text { Contusions, lacerations, } \\
\text { sprains and strains are } \\
\text { the most common } \\
\text { mechanisms of injury. } \\
\text { Knees were the most } \\
\text { common joint injured } \\
\text { followed by the thigh, } \\
\text { groin and shoulder }\end{array}$ & $\begin{array}{l}\text { Total } \\
\text { Injuries/Total AE } \\
\times 1000 \\
\text { Total AE = games } \\
\times \text { Total players on } \\
\text { ice (6) }\end{array}$ \\
\hline $\begin{array}{l}\text { Stuart, } \\
\text { Smith [21] }\end{array}$ & 1995 & $\begin{array}{l}\text { United States } \\
\text { Hockey League }\end{array}$ & $\begin{array}{l}\text { "Injury was defined as an } \\
\text { event that kept a player } \\
\text { out of practice or } \\
\text { competition for } 24 \mathrm{~h} \text {, } \\
\text { required the attention of } \\
\text { a team physician (e.g., } \\
\text { suturing lacerations) and } \\
\text { included all dental, eye } \\
\text { and nerve injuries and } \\
\text { concussions." }\end{array}$ & $\begin{array}{l}\text { Time } \\
\text { Loss/Medical } \\
\text { Attention } \\
\text { Definition }\end{array}$ & $\begin{array}{l}\text { Overall injury rate } \\
\text { was } 9.4 / 1000 \\
\text { player hours, } \\
\text { game injury rate } \\
\text { was } 96.1 / 1000 \\
\text { player hours, } \\
\text { practice injury } \\
\text { rate was } 3.9 / 1000 \\
\text { player hours }\end{array}$ & $\begin{array}{l}\text { Collision } 51 \% \text {, } \\
\text { stick contact } 14 \% \text {, } \\
\text { skate/puck contact } \\
11 \% \text {, } \\
\text { off-ice injuries } 8 \%\end{array}$ & $\begin{array}{l}\text { Strains, lacerations and } \\
\text { contusions were most } \\
\text { common mechanism. } \\
\text { Aside from the face, the } \\
\text { shoulder, hip, lumbar } \\
\text { spine and knee were } \\
\text { the most common } \\
\text { anatomical sites of } \\
\text { injury }\end{array}$ & $\begin{array}{l}\text { Total Injury/AE } \times \\
1000=\text { Practice } \\
\text { Injury Rate; } \\
\text { Practice AE }= \\
\text { Practice Hours } \times \\
\text { Roster }(25) \\
\text { Total injuries/Total } \\
\mathrm{AE} \times 1000 \text {; Total } \\
\mathrm{AE}=\text { number of } \\
\text { games } \times \text { Total } \\
\text { players on ice }(6)\end{array}$ \\
\hline $\begin{array}{l}\text { Cunningham } \\
\text { [32] }\end{array}$ & 1996 & $\begin{array}{l}\text { University } \\
\text { Games }\end{array}$ & $\begin{array}{l}\text { "A recordable injury was } \\
\text { defined as any incident } \\
\text { occurring during } \\
\text { warm-up or competition } \\
\text { and which required } \\
\text { medical attention, } \\
\text { on-field management to } \\
\text { enable continued } \\
\text { participation, or removal } \\
\text { from the playing field." }\end{array}$ & $\begin{array}{l}\text { Medical } \\
\text { Attention }\end{array}$ & $\begin{array}{l}33.5 \% \text { of injuries } \\
\text { in relation to total } \\
\text { number playing } \\
\text { the sport }\end{array}$ & Player collision & $\begin{array}{l}\text { Muscle strains and } \\
\text { hematoma }(21.7 \%)\end{array}$ & $\begin{array}{l}\text { Number of } \\
\text { injuries/number } \\
\text { of players } \\
\text { participating }\end{array}$ \\
\hline
\end{tabular}


Table 2. Cont.

\begin{tabular}{|c|c|c|c|c|c|c|c|c|}
\hline Authors & Year & Demographic & Injury Definition & Type & Injury Rate & $\begin{array}{l}\text { Mechanism of } \\
\text { Injury }\end{array}$ & Injury Type & $\begin{array}{l}\text { Injury Rate } \\
\text { Computation }\end{array}$ \\
\hline $\begin{array}{l}\text { Molsa, } \\
\text { Airaksinen, } \\
\text { Nasman, } \\
\text { Torstila [33] }\end{array}$ & 1997 & $\begin{array}{l}\text { Finnish } \\
\text { National } \\
\text { League, Finnish } \\
\text { First Division }\end{array}$ & $\begin{array}{l}\text { "An injury was defined } \\
\text { as any trauma occurring } \\
\text { during practices or games } \\
\text { and causing absence from } \\
\text { the next practice or game } \\
\text { or needing treatment (ex. } \\
\text { stitches), examination by } \\
\text { a physician (ex. } \\
\text { radiographs), or } \\
\text { rehabilitation prescribed } \\
\text { by a physician (ex. } \\
\text { physical therapy). } \\
\text { Injuries due to overuse } \\
\text { were excluded." }\end{array}$ & $\begin{array}{l}\text { Time } \\
\text { Loss/Medical } \\
\text { Attention } \\
\text { Definition }\end{array}$ & $\begin{array}{l}66 / 1000 \\
\text { player-game } \\
\text { hours, 36/1000 } \\
\text { player game } \\
\text { hours (Div. I) }\end{array}$ & $\begin{array}{l}\text { Checking } 29.7 \% \text {, } \\
\text { stick } 14.6 \% \text {, } \\
\text { contact with } \\
\text { opponent } 14.6 \% \text {, } \\
\text { puck } 7.9 \%\end{array}$ & $\begin{array}{l}\text { Contusions, strains and } \\
\text { sprains were the most } \\
\text { common type of injury, } \\
\text { the knee joint and groin } \\
\text { were the most common } \\
\text { locations }\end{array}$ & $\begin{array}{l}\text { Total Injury } / \mathrm{AE} \times \\
1000=\text { Practice } \\
\text { Injury Rate } \\
\text { Practice } \mathrm{AE}= \\
\text { Practice Hours } \times \\
\text { Roster }(21) \\
\text { Total } \\
\text { Injuries/Total AE } \\
\times 1000 \text {; } \\
\text { Total AE = } \\
\text { number of games } \\
\times \text { Total players on } \\
\text { ice }(6)\end{array}$ \\
\hline $\begin{array}{l}\text { Pinto, Kuhn, } \\
\text { Greenfield, } \\
\text { Hawkins } \\
\text { [34] }\end{array}$ & 1999 & $\begin{array}{l}\text { Junior A Hokey } \\
\text { Players (22 } \\
\text { players) }\end{array}$ & $\begin{array}{l}\text { "An injury was defined } \\
\text { as any event that } \\
\text { required the attention of } \\
\text { a physician or trainer." }\end{array}$ & $\begin{array}{l}\text { Medical } \\
\text { Attention } \\
\text { Definition }\end{array}$ & $\begin{array}{l}\text { 121/1000 player } \\
\text { game hours }\end{array}$ & $\begin{array}{l}\text { Contact with stick } \\
16.2 \% \text {, } \\
\text { overuse } 13.5 \%\end{array}$ & $\begin{array}{l}\text { Sprains/subluxations/ } \\
\text { dislocations were the } \\
\text { most common, aside } \\
\text { from the face, the } \\
\text { shoulder and knee were } \\
\text { the most common }\end{array}$ & $\begin{array}{l}\text { Total Injury/AE } \times \\
1000=\text { Practice } \\
\text { Injury Rate; } \\
\text { Practice AE = } \\
\text { Practice Hours x } \\
\text { Roster }(22) \\
\text { Total } \\
\text { Injuries/Total AE } \\
\text { x1000 } \\
\text { Total AE = } \\
\text { \#games } \times \text { Total } \\
\text { players on ice }(6)\end{array}$ \\
\hline
\end{tabular}


Table 2. Cont.

\begin{tabular}{|c|c|c|c|c|c|c|c|c|}
\hline Authors & Year & Demographic & Injury Definition & Type & Injury Rate & $\begin{array}{l}\text { Mechanism of } \\
\text { Injury }\end{array}$ & Injury Type & $\begin{array}{l}\text { Injury Rate } \\
\text { Computation }\end{array}$ \\
\hline $\begin{array}{l}\text { Molsa, } \\
\text { Kujala, } \\
\text { Nasman, } \\
\text { Lehtipuu, } \\
\text { Airaksinen } \\
\text { [35] }\end{array}$ & 2000 & $\begin{array}{l}\text { Finnish Elite } \\
\text { League }(7 \text { teams, } \\
3 \text { different } \\
\text { decades) }\end{array}$ & $\begin{array}{l}\text { "An injury was defined } \\
\text { as any sudden trauma } \\
\text { occurring during practice } \\
\text { or game that led to } \\
\text { examination and } \\
\text { treatment by a physician." } \\
\text { Minor injuries requiring } \\
\text { no absence were also } \\
\text { included, but minor } \\
\text { injuries needing no } \\
\text { medical care and injuries } \\
\text { due to overuse were } \\
\text { excluded }\end{array}$ & $\begin{array}{l}\text { Medical } \\
\text { Attention } \\
\text { Definition }\end{array}$ & $\begin{array}{l}\text { Game injury rate } \\
\text { increased from } \\
54 / 1000 \text { player } \\
\text { hours in the } 70 \text { 's } \\
\text { to } 83 / 1000 \text { player } \\
\text { hours in the } 90 \text { 's, } \\
\text { most common } \\
\text { mechanism was } \\
\text { collision }\end{array}$ & $\begin{array}{l}\text { Checking, stick, } \\
\text { falling, collision } \\
\text { with opponent, } \\
\text { puck, collision with } \\
\text { boards }\end{array}$ & $\begin{array}{l}\text { Contusions, } \\
\text { sprains/strains and } \\
\text { lacerations were the } \\
\text { most common } \\
\text { mechanisms of injury. } \\
\text { The knee was the most } \\
\text { common major injury } \\
\text { of the lower quadrant }\end{array}$ & $\begin{array}{l}\text { Player years of } \\
\text { exposure, } \\
(\text { Seasons } \times \text { Teams } \\
\times \text { Players }) \times \\
\text { Practice Hours } x \\
\text { Roster }=\text { Practice } \\
\text { Injury Rate: } \\
\text { Player years of } \\
\text { exposure, } \\
\text { (Seasons } \times \text { Teams } \\
\times \text { Players }) \times \\
\text { Game Hours } \times \\
\text { Roster }(6)=\text { Game } \\
\text { Injury Rate. }\end{array}$ \\
\hline $\begin{array}{l}\text { Flik, Lyman, } \\
\text { Marx, [19] }\end{array}$ & 2005 & $\begin{array}{l}\text { American Men's } \\
\text { Collegiate Ice } \\
\text { Hockey ( } 8 \\
\text { teams/1 season) }\end{array}$ & $\begin{array}{l}\text { "An injury was defined } \\
\text { specifically as any } \\
\text { injurious episode that led } \\
\text { to loss of participation in } \\
\text { the immediate } \\
\text { subsequent AE, whether } \\
\text { it was a practice or } \\
\text { game." }\end{array}$ & $\begin{array}{l}\text { Time Loss } \\
\text { Definition }\end{array}$ & $\begin{array}{l}\text { Overall injury rate } \\
\text { was } 4.9 / 1000 \mathrm{AE}, \\
13.8 / 1000 \mathrm{AE} \\
\text { games, } 2.2 / 1000 \\
\text { AE practice }\end{array}$ & $\begin{array}{l}\text { Collision with } \\
\text { opponent } 32.8 \% \text {, } \\
\text { collision with } \\
\text { boards } 18.6 \% \text {, } \\
\text { overuse } 8 \% \text {, } \\
\text { puck } 6.2 \%\end{array}$ & $\begin{array}{l}\text { Concussions were the } \\
\text { most common, } \\
\text { followed by knee (MCL) } \\
\text { and shoulder injuries }\end{array}$ & $\begin{array}{l}\text { Total Injury/AE } \times \\
1000=\text { Practice } \\
\text { Injury Rate } \\
\text { Practice AE }= \\
\text { Practice Hours } \times \\
\text { Roster Est } \\
\text { Total } \\
\text { Injuries/Total AE } \\
\times 1000 ; \\
\text { Total AE = } \\
\text { number of games } \\
\times \text { Total player avg } \\
\text { attendance }\end{array}$ \\
\hline
\end{tabular}


Table 2. Cont.

\begin{tabular}{|c|c|c|c|c|c|c|c|c|}
\hline Authors & Year & Demographic & Injury Definition & Type & Injury Rate & $\begin{array}{l}\text { Mechanism of } \\
\text { Injury }\end{array}$ & Injury Type & $\begin{array}{l}\text { Injury Rate } \\
\text { Computation }\end{array}$ \\
\hline $\begin{array}{l}\text { Agel, } \\
\text { Dompier, } \\
\text { Dick, } \\
\text { Marshall } \\
\text { [36] }\end{array}$ & 2007 & $\begin{array}{l}\text { NCAA Men's } \\
\text { Ice Hockey (16 } \\
\text { years of data: } \\
\text { Div. I-III) }\end{array}$ & $\begin{array}{l}\text { "A reportable injury in } \\
\text { the ISS was defined as } \\
\text { one that (1) occurred as a } \\
\text { result of participation in } \\
\text { an organized } \\
\text { intercollegiate practice or } \\
\text { competition and (2) } \\
\text { required medical } \\
\text { attention by a team } \\
\text { certified athletic trainer } \\
\text { or physician and (3) } \\
\text { resulted in restriction of } \\
\text { the student-athlete's } \\
\text { participation or } \\
\text { performance for } 1 \text { or } \\
\text { more calendar days } \\
\text { beyond the day of injury. } \\
\text { The injury definition was } \\
\text { expanded in the '94-95' } \\
\text { academic year to include } \\
\text { any dental injury } \\
\text { occurring in an organized } \\
\text { practice or game, } \\
\text { regardless of time lost." }\end{array}$ & $\begin{array}{l}\text { Time Loss } \\
\text { Definition }\end{array}$ & $\begin{array}{l}16.27 / 1000 \mathrm{AE} \\
\text { games, } 1.96 / 1000 \\
\text { AE practice }\end{array}$ & $\begin{array}{l}\text { Player contact } 50 \% \text {, } \\
\text { other contact } 39.6 \% \text {, } \\
\text { no contact } 9.7 \% \\
\text { (game numbers). } \\
\text { Injury was } 8 \mathrm{x} \\
\text { higher in games. }\end{array}$ & $\begin{array}{l}\text { Knee internal } \\
\text { derangement }(13.5 \%) \\
\text { was the most common } \\
\text { lower extremity injury } \\
\text { reported during games, } \\
\text { followed by } \\
\text { concussions and AC } \\
\text { injuries. Whereas } \\
\text { pelvis and hip strains } \\
(13.1 \%) \text { were the most } \\
\text { common injury } \\
\text { reported at practice. }\end{array}$ & $\begin{array}{l}\text { Total Injury } / \mathrm{AE} \times \\
1000=\text { Practice } \\
\text { Injury Rate; } \\
\text { Practice AE }= \\
\text { Practice Hours } \times \\
\text { Roster }(26) \\
\text { Total } \\
\text { Injuries/Total AE } \\
\times 1000 \\
\text { Total AE = } \\
\text { number of games } \\
\times \text { Total players } \\
(19)\end{array}$ \\
\hline
\end{tabular}


Table 2. Cont.

\begin{tabular}{|c|c|c|c|c|c|c|c|c|}
\hline Authors & Year & Demographic & Injury Definition & Type & Injury Rate & $\begin{array}{l}\text { Mechanism of } \\
\text { Injury }\end{array}$ & Injury Type & $\begin{array}{l}\text { Injury Rate } \\
\text { Computation }\end{array}$ \\
\hline $\begin{array}{l}\text { Rishiraj, } \\
\text { Lloyd-Smith, } \\
\text { Lorenz, } \\
\text { Michel [37] }\end{array}$ & 2009 & $\begin{array}{l}\text { Men's Varsity } \\
\text { Ice Hockey } \\
\text { (Canada) }\end{array}$ & $\begin{array}{l}\text { "Any event, during team } \\
\text { or team related game, } \\
\text { practice, and/or activity } \\
\text { (on or off the ice), } \\
\text { requiring any attention } \\
\text { by the team's therapist } \\
\text { and/or physician and } \\
\text { subsequent game and/or } \\
\text { practice time loss." }\end{array}$ & $\begin{array}{l}\text { Time Loss } \\
\text { Definition }\end{array}$ & $\begin{array}{l}\text { 3.7/1000 player } \\
\text { game and practice } \\
\text { exposure }\end{array}$ & $\begin{array}{l}\text { Non-contact, } \\
\text { ice/board contact, } \\
\text { body contact }\end{array}$ & $\begin{array}{l}\text { Sprains } 20 \% \text {, strains } \\
20 \% \text {, concussions } 13 \% \\
\text { and contusions } 12 \%\end{array}$ & $\begin{array}{l}\text { Total Injury/AE } \times \\
\text { 1000; } \\
\text { Practice AE }= \\
\text { Practice Hours } \times \\
\text { Roster } \\
\text { Total } \\
\text { Injuries/Total AE } \\
\text { x1000 } \\
\text { Total AE }= \\
\text { number of games } \\
\times \text { Roster Selected }\end{array}$ \\
\hline $\begin{array}{l}\text { Kuzuhara, } \\
\text { Shimamoto, } \\
\text { Mase [38] }\end{array}$ & 2009 & $\begin{array}{l}\text { Japanese Elite } \\
\text { Team }\end{array}$ & $\begin{array}{l}\text { "An injury was defined } \\
\text { as any event that } \\
\text { occurred during on-ice } \\
\text { practices or games that } \\
\text { required medical } \\
\text { attention and treatment." }\end{array}$ & $\begin{array}{l}\text { Medical } \\
\text { Attention } \\
\text { Definition }\end{array}$ & $\begin{array}{l}74.3 / 1000 \text { player } \\
\text { game hours, } \\
11.7 / 1000 \\
\text { player-game } \\
\text { hours for injuries } \\
\text { resulting in any } \\
\text { time loss, } \\
11.2 / 1000 \\
\text { player-practice } \\
\text { hours, } 1.1 / 1000 \\
\text { player-practice } \\
\text { hours for injuries } \\
\text { resulting in any } \\
\text { time loss }\end{array}$ & $\begin{array}{l}\text { Overuse } 52 \% \text {, } \\
\text { puck contact } 21 \% \text {, } \\
\text { stick contact } 15 \% \text {, } \\
\text { falling } 12 \%\end{array}$ & $\begin{array}{l}\text { Contusions } 35.4 \% \text {, } \\
\text { strains } 15.6 \% \text {, } \\
\text { lacerations } 9.3 \%\end{array}$ & $\begin{array}{l}\begin{array}{l}\text { Overall injury rate } \\
\text { (regardless of time }\end{array} \\
\text { loss): \#of } \\
\text { injuries/number } \\
\text { of hours per } 1000 \\
\text { player-hours } \\
\text { number of injuries } \\
\text { causing time loss } \\
\text { ( } 1 \text { day)/number } \\
\text { of hours per } 1000 \\
\text { player-hours } \\
\text { Number of player: } \\
2003 \text { (20 } \\
\text { players/game, 25 } \\
\text { players/practice), } \\
\text { 2004 (20 } \\
\text { players/game, } \\
\text { 37players/practice) } \\
\text { 2005 (22 } \\
\text { players/game, 32 } \\
\text { players/practice) }\end{array}$ \\
\hline
\end{tabular}


Table 2. Cont.

\begin{tabular}{|c|c|c|c|c|c|c|c|c|}
\hline Authors & Year & Demographic & Injury Definition & Type & Injury Rate & $\begin{array}{c}\text { Mechanism of } \\
\text { Injury }\end{array}$ & Injury Type & $\begin{array}{c}\text { Injury Rate } \\
\text { Computation }\end{array}$ \\
\hline $\begin{array}{l}\text { Agel, } \\
\text { Harvey [39] }\end{array}$ & 2010 & $\begin{array}{l}\text { NCAA Men's } \\
\text { and Women's } \\
\text { Ice Hockey (Div. } \\
\text { I and III) }\end{array}$ & Same as Dick et al. above & $\begin{array}{l}\text { Time Loss } \\
\text { Definition }\end{array}$ & $\begin{array}{l}\text { 18.69/1000 AE } \\
\text { games, } 2.23 / 1000 \\
\text { AE practice for } \\
\text { men, } \\
12.10 / 1000 \mathrm{AE} \\
\text { games, } 2.90 / 1000 \\
\text { AE practice for } \\
\text { women }\end{array}$ & Player contact $48 \%$ & $\begin{array}{l}\text { The most common } \\
\text { injury among men was } \\
\text { concussion followed by } \\
\text { shoulder and knee } \\
\text { ligamentous in juries }\end{array}$ & $\begin{array}{l}\text { Number of } \\
\text { Injuries/Number } \\
\text { of AE }(\text { games or } \\
\text { practice } \times \text { roster } \\
\times 1000\end{array}$ \\
\hline $\begin{array}{l}\text { Engebretsen, } \\
\text { Steffen, } \\
\text { Alonso, } \\
\text { Dvorak, } \\
\text { Junge, } \\
\text { Meeuwisse, } \\
\text { Mountjoy, } \\
\text { Renstrom, } \\
\text { Wlikinson } \\
\text { [40] }\end{array}$ & 2010 & Olympic Sport & $\begin{array}{l}\text { "An athlete was defined } \\
\text { as injured or ill if he/she } \\
\text { received medical } \\
\text { attention regardless of } \\
\text { the consequences with } \\
\text { respect to absence from } \\
\text { competition or training." }\end{array}$ & $\begin{array}{l}\text { Medical } \\
\text { Attention } \\
\text { Definition }\end{array}$ & $\begin{array}{l}\text { A total injury rate } \\
\text { of } 111.8 / 1000 \mathrm{AE} \\
\text { was reported for } \\
\text { both males and } \\
\text { females. A total of } \\
276 \text { males were } \\
\text { registered with } 44 \\
\text { total injuries ( } 16 \%) \\
\text { in men's elite ice } \\
\text { hockey. }\end{array}$ & N/A & N/A & $\begin{array}{l}\text { Number of } \\
\text { Injuries/Athlete } \\
\text { Exposure }\end{array}$ \\
\hline
\end{tabular}


Table 2. Cont.

\begin{tabular}{|c|c|c|c|c|c|c|c|}
\hline Authors & Year & Demographic & Injury Definition & Injury Rate & $\begin{array}{c}\text { Mechanism of } \\
\text { Injury }\end{array}$ & Injury Type & $\begin{array}{l}\text { Injury Rate } \\
\text { Computation }\end{array}$ \\
\hline $\begin{array}{l}\text { Kerr, } \\
\text { Dompier, } \\
\text { Snook, } \\
\text { Marshall, } \\
\text { Klossner, } \\
\text { Hainline, } \\
\text { Corlette [41] }\end{array}$ & 2014 & NCAA Sports & $\begin{array}{l}\text { "Any injury occurring } \\
\text { during an organized } \\
\text { intercollegiate practice or } \\
\text { game." (1982) "A } \\
\text { reportable injury was } \\
\text { defined as an injury that } \\
\text { (1) occurred as a result of } \\
\text { participation in an } \\
\text { organized intercollegiate } \\
\text { practice or competition, } \\
\text { (2) required attention } \\
\text { from an AT or physician, } \\
\text { and (3) resulted in } \\
\text { restriction of the } \\
\text { student-athlete's } \\
\text { participation for } 1 \text { or } \\
\text { more days beyond the } \\
\text { day of injury." Multiple } \\
\text { injuries from one event } \\
\text { could be included. In } \\
\text { addition, AT's were asked } \\
\text { to include any dental } \\
\text { injuries that occurred in } \\
\text { an organized practice or } \\
\text { game, regardless of time } \\
\text { lost. 2003-onward). } \\
\text { Beginning in 2009-2010 } \\
\text { academic year, non-time } \\
\text { loss injuries were also } \\
\text { monitored. }\end{array}$ & $\begin{array}{l}\text { Time } \\
\text { Loss/Medical N/A } \\
\text { Attention } \\
\text { Definition }\end{array}$ & $\mathrm{N} / \mathrm{A}$ & $\mathrm{N} / \mathrm{A}$ & $\begin{array}{l}\text { Number of } \\
\text { Injuries/AE } \\
\text { (average team } \\
\text { roster) } \times 1000\end{array}$ \\
\hline
\end{tabular}


Table 2. Cont.

\begin{tabular}{|c|c|c|c|c|c|c|c|c|}
\hline Authors & Year & Demographic & Injury Definition & Type & Injury Rate & $\begin{array}{l}\text { Mechanism of } \\
\text { Injury }\end{array}$ & Injury Type & $\begin{array}{l}\text { Injury Rate } \\
\text { Computation }\end{array}$ \\
\hline $\begin{array}{l}\text { McKay, } \\
\text { Tufts, } \\
\text { Shaffer, } \\
\text { Meeuwisse } \\
\text { [4] }\end{array}$ & 2014 & $\begin{array}{l}\text { NHL Players } \\
(2006-2012)\end{array}$ & $\begin{array}{l}\text { "Any event captured by } \\
\text { the IIE form, and } \\
\text { restricted to those } \\
\text { designated as } \\
\text { practice-related or game } \\
\text { related, resulting in one } \\
\text { or more-man games lost. }\end{array}$ & $\begin{array}{l}\text { Time Loss } \\
\text { Definition }\end{array}$ & $\begin{array}{l}\text { 15.6/1000 AE } \\
\text { based on } \\
\text { estimated AE's. } \\
\text { Based on recorded } \\
\text { TOI *, the injury } \\
\text { rates were } \\
\text { roughly threefold } \\
\text { higher at } \\
49.4 / 1000 \\
\text { player-game } \\
\text { hours }\end{array}$ & $\begin{array}{l}\text { Body checking was } \\
\text { the most common } \\
\text { mechanism }\end{array}$ & $\begin{array}{l}\text { Most commonly injured } \\
\text { body regions were the } \\
\text { head }(16.8 \%) \text {, thigh } \\
(14 \%) \text {, and knee }(13 \%)\end{array}$ & $\begin{array}{l}\text { Estimated AEs = } \\
82 \text { games } \times 19 \\
\text { players (including } \\
\text { goalie) TOI } \\
(\text { NHL.com) }= \\
\text { number of injury } \\
\text { events/sum of } \\
\text { individual AE } \\
\text { time }\end{array}$ \\
\hline $\begin{array}{l}\text { Tuominen, } \\
\text { Stuart, } \\
\text { Aubry, } \\
\text { Kannus } \\
\text { Parkkari [42] }\end{array}$ & 2015 & $\begin{array}{l}\text { Men's } \\
\text { International Ice } \\
\text { Hockey } \\
(2006-2013)\end{array}$ & $\begin{array}{l}\text { "The definition of an } \\
\text { injury was made in } \\
\text { accordance with the } \\
\text { accepted international ice } \\
\text { hockey norms: (1) Any } \\
\text { injury sustained in a } \\
\text { practice or a game that } \\
\text { prevented the player } \\
\text { from returning to the } \\
\text { same practice or game, (2) } \\
\text { any injury sustained in a } \\
\text { practice or a game that } \\
\text { caused the player to miss } \\
\text { a subsequent practice or } \\
\text { game, (3) a laceration that } \\
\text { required medical } \\
\text { attention, (4) all dental } \\
\text { injuries, (5) all } \\
\text { concussions, (6) all } \\
\text { fractures }\end{array}$ & $\begin{array}{l}\text { Time } \\
\text { Loss/Medical } \\
\text { Attention } \\
\text { Definition }\end{array}$ & $\begin{array}{l}\text { 14.2/1000 AE } \\
\text { player games, } \\
52.1 / 1000 \mathrm{AE} \\
\text { player game } \\
\text { hours } \\
\text { For WC A-pool } \\
\text { tournaments and } \\
\text { Olympic games } \\
\text { the injury rate } \\
\text { was } 16.3 / 1000 \\
\text { player-games, } \\
69.6 / 1000 \\
\text { player-game } \\
\text { hours }\end{array}$ & $\begin{array}{l}\text { Body contact and } \\
\text { puck contact were } \\
\text { the mechanisms }\end{array}$ & $\begin{array}{l}\text { Most common types of } \\
\text { injuries were } \\
\text { lacerations, sprains, } \\
\text { strains, and contusions. } \\
\text { The knee was the most } \\
\text { commonly injured } \\
\text { lower body segment, } \\
\text { MCL was the most } \\
\text { common, and the } \\
\text { shoulder was the most } \\
\text { common site of an } \\
\text { upper body injury. }\end{array}$ & $\begin{array}{l}\text { Player game } \\
\text { injury rate (based } \\
\text { on } 22 \text { players on } \\
\text { each team): } \\
\text { Number of } \\
\text { injuries/number } \\
\text { of players (two } \\
\text { teams)/number of } \\
\text { games } \times 1000 \text {, } \\
\text { Player game-hour } \\
\text { injury rate (based } \\
\text { on } 6 \text { players on ice } \\
\text { at once): } \\
\text { number of } \\
\text { injuries/number } \\
\text { of players on ice } \\
\text { at the same time } \\
\text { (two } \\
\text { teams)/number of } \\
\text { games } \times 1000\end{array}$ \\
\hline
\end{tabular}


Table 2. Cont

\begin{tabular}{|c|c|c|c|c|c|c|c|c|}
\hline Authors & Year & Demographic & Injury Definition & Type & Injury Rate & $\begin{array}{l}\text { Mechanism of } \\
\text { Injury }\end{array}$ & Injury Type & $\begin{array}{c}\text { Injury Rate } \\
\text { Computation }\end{array}$ \\
\hline $\begin{array}{l}\text { Kerr et al. } \\
\text { [43] }\end{array}$ & 2015 & $\begin{array}{l}\text { NCAA Ice } \\
\text { Hockey }\end{array}$ & $\begin{array}{l}\text { Injuries were defined as } \\
\text { those that occurred in an } \\
\text { organized } \\
\text { NCAA-approved practice } \\
\text { or competition and } \\
\text { required medical } \\
\text { attention by a physician } \\
\text { or athletic trainer. An } \\
\text { athlete-exposure was } \\
\text { defined as one } \\
\text { student-athlete's } \\
\text { participation in one } \\
\text { practice or one } \\
\text { competition. }\end{array}$ & $\begin{array}{l}\text { Medical } \\
\text { Attention } \\
\text { Definition }\end{array}$ & 9.5/1000 AE & N/A & $\begin{array}{l}\text { Concussions, } \\
\text { contusions, fractures }\end{array}$ & $\begin{array}{l}\text { Number of } \\
\text { Injuries/Number } \\
\text { of Athlete } \\
\text { Exposures }\end{array}$ \\
\hline $\begin{array}{l}\text { Tuominen, } \\
\text { Stuart, } \\
\text { Aubry, } \\
\text { Kannus, } \\
\text { Parkkari [22] }\end{array}$ & 2016 & $\begin{array}{l}\text { World Junior } \\
\text { Hockey Players } \\
\text { (ages 18-20) }\end{array}$ & $\begin{array}{l}\text { "The definition of an } \\
\text { injury was made in } \\
\text { accordance with the } \\
\text { accepted international ice } \\
\text { hockey norms: (1) Any } \\
\text { injury sustained in a } \\
\text { practice or a game that } \\
\text { prevented the player } \\
\text { from returning to the } \\
\text { same practice or game, (2) } \\
\text { any injury sustained in a } \\
\text { practice or a game that } \\
\text { caused the player to miss } \\
\text { a subsequent practice or } \\
\text { game, (3) a laceration that } \\
\text { required medical } \\
\text { attention, (4) all dental } \\
\text { injuries, (5) all } \\
\text { concussions, (6) all } \\
\text { fractures }\end{array}$ & $\begin{array}{l}\text { Time } \\
\text { Loss/Medical } \\
\text { Attention } \\
\text { Definition }\end{array}$ & $\begin{array}{l}\text { 11/1000 AE } \\
\text { player-games, } \\
39.8 / 1000 \\
\text { player-game } \\
\text { hours }\end{array}$ & $\begin{array}{l}\text { Body checking } 32 \% \text {, } \\
\text { stick } 13 \% \text {, } \\
\text { and puck contact } \\
13 \%\end{array}$ & $\begin{array}{l}\text { The knee was the most } \\
\text { frequent site of lower } \\
\text { body injury in WJ and } \\
\text { U20 tournaments ( } 33 \%) \text {, } \\
\text { MCL sprain was most } \\
\text { common, the shoulder } \\
\text { was the most common } \\
\text { upper body injury. }\end{array}$ & $\begin{array}{l}\text { Player game } \\
\text { injury rate (based } \\
\text { on } 20-22 \text { players } \\
\text { on each team): } \\
\text { number of } \\
\text { injuries/number } \\
\text { of players (two } \\
\text { teams)/number of } \\
\text { games } \times 1000 \text {, } \\
\text { Player game-hour } \\
\text { injury rate (based } \\
\text { on } 6 \text { players on ice } \\
\text { at once): number } \\
\text { of injuries/number } \\
\text { of players on ice } \\
\text { at the same time } \\
\text { (two } \\
\text { teams)/number of } \\
\text { games } \times 1000\end{array}$ \\
\hline
\end{tabular}


Table 2. Cont.

\begin{tabular}{|c|c|c|c|c|c|c|c|}
\hline Authors & Year & Demographic & Injury Definition & Injury Rate & $\begin{array}{l}\text { Mechanism of } \\
\text { Injury }\end{array}$ & Injury Type & $\begin{array}{l}\text { Injury Rate } \\
\text { Computation }\end{array}$ \\
\hline $\begin{array}{l}\text { Lynall, } \\
\text { Mihalik, } \\
\text { Pierpoint, } \\
\text { Currie, } \\
\text { Knowles, } \\
\text { Wasserman, } \\
\text { Dompier, } \\
\text { Comstock, } \\
\text { Marshall, } \\
\text { Kerr [44] }\end{array}$ & 2018 & $\begin{array}{l}\text { Collegiate } \\
\text { Men's and } \\
\text { Women's } \\
\text { Hockey } \\
(2004-2005, \\
2013-2014)\end{array}$ & $\begin{array}{l}\text { "An injury that (1) } \\
\text { occurred as a result of } \\
\text { participation in an } \\
\text { organized practice or } \\
\text { competition; (2) required } \\
\text { medical attention by a } \\
\text { certified AT or physician; } \\
\text { and (3) resulted in } \\
\text { restriction of the } \\
\text { student-athlete's } \\
\text { participation for } 1 \text { or } \\
\text { more days beyond the } \\
\text { day of injury. Since the } \\
\text { 2007-2008 academic year, } \\
\text { HS RIO has also captured } \\
\text { all concussions, fractures, } \\
\text { and dental injuries, } \\
\text { regardless of time loss." } \\
\text { "Beginning in the } \\
\text { 2009-2010 academic year, } \\
\text { the NCAA-ISP also began } \\
\text { to monitor all } \\
\text { non-time-loss injuries. A } \\
\text { non-time-loss injury was } \\
\text { defined as any injury that } \\
\text { was evaluated or treated } \\
\text { (or both) by an AT or } \\
\text { physician but did not } \\
\text { result in restriction from } \\
\text { participation beyond the } \\
\text { day of injury." }\end{array}$ & $\begin{array}{l}\text { Medical } \\
\text { Attention/TimCollegiate Men: } \\
\text { Loss } \quad 13.45 / 1000 \mathrm{AE} \\
\text { Definition }\end{array}$ & Collision & Strains/Sprains & $\begin{array}{l}\text { Total } \\
\text { Injuries/Total } \\
\text { Athlete Exposure }\end{array}$ \\
\hline
\end{tabular}

TOI $^{*}=$ Time on Ice. ${ }^{* *}=$ Author did not specify how injury rate was calculated. 
The injury rates in practice are much lower than games. Practice rates range between 1.4/1000 player-practice hours for Swedish Elite hockey [30] to 3.9/1000 player-practice hours for junior hockey [21], versus game injury rates of 74.3/1000 player-game hours [38] and 121/1000 player-game hours [34], respectively. Although the injury rates are lower for practices, the number of hours spent in practices is several-fold greater than games, so the actual number of injuries is higher than indicated by the injury rate.

Several long-term studies have assessed patterns in injury rates over time. For example, injury rates in the Finnish Elite League have increased from the 1970s (54/1000 AE) to the 1990s (83/1000 AE) using the player-game hours exposure estimate [35] (20 years). Overall game injury rates increased $1.8 \%$ annually over a seven-year period (2000-2007) in men's NCAA ice hockey using the player game estimate. Practice rates also increased $7.8 \%$ annually during this time [39]. In contrast, on average, injury rates have decreased between 2007 and 2013 in men's International Ice Hockey Federation World Championship tournaments [42] (6-years). One Canadian Intercollegiate team also experienced decreases in injury rate over a six-year period from 11.3 to 8.30/1000 player games (1991-1996) [37] (6-years).

There was a large variance in injury rates between studies. This large variance is a function of variability in the definitions for both injury and athlete exposure. As noted in previous papers, establishing consistent definitions of injury and athlete exposure are important first steps for objectifying injury risks in high caliber ice hockey $[10,45]$.

\subsection{Injury Definition in Men's Elite Ice Hockey (Question \#2)}

Probably the most important methodological factor affecting injury rate calculations is the definition of what constitutes an injury [45]. A review investigating the methods of data collection on injury surveillance identified three categories of injury definitions [45]. Category 1 defines injuries as all complaints regardless of time loss. All injuries are recorded, regardless of the severity or amount of time lost from competition. Category 2 defines injuries as events that require medical attention by a member of the medical staff. Therefore, according to this definition, a member of the medical staff, typically a team therapist or team doctor, must diagnose the injury. Finally, category 3 defines injuries as events that have a time loss element. Accordingly, an injury is only recorded if the athlete misses a team-related practice or competition. Individual studies typically fit into one, or more of these categories.

Our review identified 28 studies evaluating injuries in elite ice hockey. Early research investigating injury rates in the Swedish Elite League, and the Swedish National team used the time loss definition of injury (Category 3). As shown in Table 2, the majority of ice hockey injuries studies use either a time loss (Category 3) or medical attention definition (Category 2). None of the articles evaluating injuries in elite ice hockey used the all complaints definition (Category 1).

Our review found inconsistent definitions of a reportable injury in ice hockey research based on the time loss definition. In addition, the list of injuries has expanded over time. Facial lacerations were considered reportable injuries in 1991 [24], while sutures, fractures, dislocations and subluxations were added in 1992 [23]. Concussions, dental and eye injuries were added in subsequent years [20,21], potentially increasing injury rates by expanding the list of injuries. In addition, illness may be counted as an injury, inflating the injury rates [23].

The definition of injury based on medical attention (Category 2) has also been used to quantify competitive ice hockey injury rates $[31,34,38]$. However, this metric is often combined with the time loss component to result in a broader interpretation of injuries [20-23,35,42]. For example, injuries such as concussions, dental injuries, lacerations and eye injuries are captured with medical attention by a team physician or athletic trainer, resulting in a more extensive list of ice hockey related injuries compared to definitions that did not include these injuries [45]. Of note, some studies have expanded their list to include illnesses and psychological complaints that are unrelated to injury [46]. 
The time loss definition (Category 3 ) is the easiest to use as it is easy to track time loss. However, it leads to the fewest reported incidents [45] as it fails to capture the athletes that continue to train and play while injured [47]. Depending on the time of year, some injuries may be under reported as injured players continue to play throughout key time periods, such as playoffs. The medical attention definition (Category 2), though broader and encompassing a greater number of conditions, also has limitations. The subjective interpretation of what constitutes medical attention may lead to systemic bias [48], and the types of injuries managed by the various practitioners may differ based on their qualifications and status [45].

\subsection{Athlete Exposure Metric in Men's Elite Ice Hockey (Question \#3)}

Athlete exposure is the second component of injury rate. An athlete exposure is defined as one athlete participating in a practice or game in which there is a potential for athletic injury [49]. Injury rates are typically based on 1000 athlete exposures. These exposure rates can be quantified as injuries per 1000 game-hours (or injuries per 1000 games), injuries per 1000 practice-hours, or overall injuries per $1000 \mathrm{AEs}$ (games and practices combined). Injury per 1000 player-game hours is based on a 60-min active game and is calculated as the number of injuries/number of players on the ice at the same time (6)/number of games $\times 1000$. Many researchers use this method $[18,21,24,30,31,33,34]$. However, this exposure estimate is not used consistently among researchers. For example, several studies accounted for both teams when calculating athlete exposure (number of injuries/number of players on ice at the same time (two teams)/number of games $\times 1000$ [22,42]. In contrast, another study used a 20 person roster, including the back-up goaltender, to calculate athlete exposure per 1000 player-game hours [38]. This larger number of players will lead to a smaller injury rate.

Our review identified different nomenclatures pertaining to the athlete exposure metric, such as player-games and player-game hours [42]. The number of athletes used to quantify these exposure rates vary between studies, and are not consistently defined. For example, one researcher [42] calculated player-game injury rates based on 22 players competing for each team in a game (i.e., 44 players) while another [30] calculated player-game hours injury rates based on 6 players. This was based on the number of players on the ice at a time, and whether goaltenders were included. Other researchers have used roster averages over a set period of time [36,37], or a tournament [22,42] to calculate player-game injury rates.

Injury per 1000 games is the average number of injuries that one player experiences per 1000 games (number of injuries/total number of players (roster)/number of games $\times 1000$ [20,37]. Our review found different implementations of this approach as there was some research that counted both rosters when computing athlete exposure [42]. This has an effect on total estimated exposures and can lead to reduced injury rates. Finally, several articles did not fully describe whether they included both rosters or a single team roster when calculating athlete exposures $[19,23]$, making it difficult to determine accurate injury rates.

In addition, we investigated the impact of calculating injury rate based on the actual time on ice (TOI) $[4,50]$. Using the actual time on ice, injury rate was calculated as the number of injury events/sum of individual AE time as found on the player statistics page (www.nhl.com/stats/player). The time on ice was calculated based on the number of minutes and seconds that each individual played per game over the season. The difference between estimated athlete exposure (number of injuries/number of teams (30)/number of players on roster each game (19)/number of games (82)) and the TOI metric was large. As much as three times the amount of exposure was identified by estimating exposure rates. However, when comparing the time on ice metric to the estimated player game-hour metric, the differences were minimal. The player game-hour exposure (based on one hour per game rather than the actual amount of time that players spent on ice, which changes due to overtime periods and penalties) is similar to the time on ice calculations $(14,676.2 \mathrm{~h}$ calculated as the sum of players' time on ice versus $14,760 \mathrm{~h}$ calculated as 30 teams $\times 82$ games $\times 6$ players) [4]. 
Our review found that practice athlete exposure was calculated consistently in most studies. Injury per 1000 practice hours (number of injuries/number of practice hours/number of players on team $\times 1000)$ was the standard $[21,30,33,34]$.

\section{Discussion}

Injury rates in men's elite ice hockey are higher in professional leagues such as the Swedish Elite League [31] and Finnish National League [33] than college hockey [19,20,23]. This may be due to the differing demands as professional players play more games in a season, and therefore may experience more overuse injuries. It may also be due to the athlete exposure estimation (player-game hours vs. player-games) used to calculate injury rate. Style of play and hockey rink dimensions are additional variables that may influence injury rate. Overall, we observed the trend that injury rates have increased over time in professional European leagues [35] and college hockey [39], while decreasing in men's international ice hockey [42].

We observed a wide range of injury definitions. This affects both the reliability and comparability of injury surveillance research. There is currently a consensus-based injury definition in sports such as soccer and rugby $[10,11]$; however, there is no consensus injury definition in ice hockey. We recommend that hockey forms a consensus injury definition as this will resolve an important issue that currently impedes hockey injury research. A consistent injury definition would create clarity as to which injury is considered a recordable event. We identified the International Ice Hockey Federation's (IIHF) definition of injury as the most appropriate as it only captures events that are sufficiently severe that they influence participation in practices or games. The IIHF's definition describes a reportable event as "any injury sustained in a practice or game that prevented the player from returning to the same practice or game; any injury sustained in a practice or game that caused the player to miss a subsequent practice or game; a laceration which required medical attention; all dental injuries; all concussions; all fractures" [42]. Although no single definition suits all needs, the time loss definition is the most common and easy to identify. It is considered reliable and allows for the comparison of data between teams, seasons and various leagues [45]. It is also used in other professional sports such as cricket and Australian football [51,52]. The choice of definition should reflect the aims and goals of surveillance. With its consistency, ease of use, and comparability of published data [8] among the most important variables, we feel the time-loss definition best meets the needs of injury surveillance in men's elite ice hockey. However, like all definitions there are limitations in choosing this metric. First, athletes often continue to compete in the presence of injury. Delaying treatment may lead to missed injuries. Finally, the threshold for time loss may depend on the time of season and how important the player is to team success [45]. Despite these drawbacks, we feel the strengths of the time-loss definition outweigh its limitations and that the IIHF's time-loss definition is warranted in elite men's ice hockey.

We also noted that athlete exposure estimations were inconsistent in the literature. The major confusion lies in how many participants are included in the injury rate calculation. Several researchers used player-game exposure based on the entire team, or average team roster (19 players) [20,36,37], while others used player-game hour exposures based on 6 players $[18,21,24,30,34]$. This leads to difficulty in interpreting injury rates and comparing research. It was proposed that the gold standard in athlete exposure during games is time on ice. As much as three times the amount of exposure was accounted for by estimating exposure rates using the player-game approximation compared to time on ice. However, when comparing the time on ice metric to the estimated player game-hour metric (based on one hour per game, rather than the actual amount of time that players spent on ice) it appears that this difference is small [4]. Therefore, the simplest and easiest way to calculate athlete exposure is to use six players on the ice (player-game hours) unless position specific injury rate information is warranted. Using a consistent athlete exposure metric will increase intra- and inter-league injury rate reliability.

The majority of studies reviewed found that collision with other players is the leading mechanism of injury as well as contact with the boards, opponent's hockey sticks and hockey pucks $[22,35,36]$. 
This leads to an injury paradox: the goal of the sports performance specialist is to build bigger, faster, stronger, leaner, more powerful, robust players. However, these types of players also travel faster, and hit harder, elevating the risk of injury. This situation emphasizes the need for accurate injury surveillance methods as these may help reinforce rules and/or govern the addition of new rules enforcing safety for active players.

\section{Limitations}

There are limitations to this study. There is a relative paucity of studies evaluating injury rates in men's elite ice hockey, and the definitions of injury and athlete exposures vary between studies. Accordingly, the reported injury rates differ between studies and are difficult to interpret. Two databases (PubMed and SPORTDiscus) were used to identify research papers that were relevant to injury definition, injury rates and athlete exposure in elite ice hockey. While these databases are an excellent source for research articles in sports, life sciences and biomedicine, supplemental databases may have identified additional research studies.

\section{Conclusions}

In summary, this project represents the first integrative literature review investigating injury rates, injury definition and AE in men's elite ice hockey. The greatest opportunities for continued improvement lie in both consistency and comparability to refine, improve and streamline calculations of injury rate.

At the current moment, a uniform definition of injury is the most important step to better objectify injury data in ice hockey. A universal definition is required by sport governing bodies and researchers. Though each approach has its limitations, in order to compare exposure rates in both the intra- and inter-league, a workable, consistent definition is required. Specific responsibility should be given in terms of who will diagnose the injury if the definition is a time loss definition, a medical attention definition, or a combination. In addition, a detailed injury list is needed to clarify the definition of injury and whether specific injuries such as dental, concussions, and facial lacerations, are included.

Finally, disparate AE estimations diminish injury rates, which compromises research findings. Attendance rate in both practice and games (player-game hours based on 6 players per game and the full roster during practices) is the preferred method for calculating athlete exposure.

\section{Further Research}

Investigating anatomical areas prone to injury is crucial for team performance staff such as athletic therapists, physical therapists and strength and conditioning specialists as it may guide rehabilitation initiatives, performance program design and athlete monitoring [53]. We observed that the lower extremities was the most common site of musculoskeletal injury.

Future research should clearly define injury rate measurements to provide doctors, therapists, and coaches with accurate information to streamline return to play initiatives. In this regard, our review has exposed gaps including the disparate definition of injury and the lack of a consistent athlete exposure metric.

Author Contributions: Study conceptualization, A.S.D.; methodology, A.S.D.; formal analysis, original draft preparation, A.S.D.; writing—review and editing, A.S.D., D.H. and J.P.D.; project administration, J.P.D.

Funding: This research received no external funding.

Acknowledgments: The authors thank David Lesauvage, Library Assistant, University of Western Ontario, Canada, for his contribution in refining a comprehensive search strategy for our review. The authors declare they have no competing interest. The study complied with the laws of the country of the authors' affiliation.

Conflicts of Interest: The authors declare no conflict of interest. 


\section{References}

1. Sim, F.H.; Simonet, W.T.; Melton, L.J., 3rd; Lehn, T.A. Ice hockey injuries. Am. J. Sports Med. 1987, 15, 30-40. [CrossRef] [PubMed]

2. Caputo, P.; Mattson, D.J. Recreational ice hockey injuries in adult non-checking leagues: A United States perspective. J. Sports Sci. Med. 2005, 4, 58-65. [PubMed]

3. Emery, C.A.; Kang, J.; Shrier, I.; Goulet, C.; Hagel, B.E.; Benson, B.W.; Nettel-Aguirre, A.; McAllister, J.R.; Hamilton, G.M.; Meeuwisse, W.H. Risk of injury associated with body checking among youth ice hockey players. JAMA 2010, 303, 2265-2272. [CrossRef] [PubMed]

4. McKay, C.D.; Tufts, R.J.; Shaffer, B.; Meeuwisse, W.H. The epidemiology of professional ice hockey injuries: A prospective report of six NHL seasons. Br. J. Sports Med. 2014, 48, 57-62. [CrossRef] [PubMed]

5. Donaldson, L.; Li, B.; Cusimano, M.D. Economic burden of time lost due to injury in NHL hockey players. Inj. Prev. 2014, 20, 347-349. [CrossRef] [PubMed]

6. Hodgson, L.; Gissane, C.; Gabbett, T.J.; King, D.A. For debate: Consensus injury definitions in team sports should focus on encompassing all injuries. Clin. J. Sport Med. 2007, 17, 188-191. [CrossRef]

7. Wennberg, R. Effect of ice surface size on collision rates and head impacts at the World Junior Hockey Championships, 2002 to 2004. Clin. J. Sport Med. 2005, 15, 67-72. [CrossRef]

8. Finch, C.F. An overview of some definitional issues for sports injury surveillance. Sports Med. 1997, 24, 157-163. [CrossRef]

9. Cropper, E.; Thorpe, C.M.; Roberts, S.; Twist, C. Injury Surveillance during a European Touch Rugby Championship. Sports 2019, 7, 71. [CrossRef]

10. Fuller, C.W.; Ekstrand, J.; Junge, A.; Andersen, T.E.; Bahr, R.; Dvorak, J.; Hägglund, M.; McCrory, P.; Meeuwisse, W.H. Consensus statement on injury definitions and data collection procedures in studies of football (soccer) injuries. Scand. J. Med. Sci. Sports 2006, 16, 83-92. [CrossRef]

11. Fuller, C.W.; Molloy, M.G.; Bagate, C.; Bahr, R.; Brooks, J.H.; Donson, H.; Kemp, S.P.; McCrory, P.; McIntosh, A.S.; Meeuwisse, W.H. Consensus statement on injury definitions and data collection procedures for studies of injuries in rugby union. Br. J. Sports Med. 2007, 41, 328-331. [CrossRef]

12. Ekstrand, J.; Karlsson, J. The risk for injury in football. There is a need for a consensus about definition of the injury and the design of studies. Scand. J. Med. Sci. Sports 2003, 13, 147-149. [CrossRef]

13. Crowley, S.G.; Trofa, D.P.; Vosseller, J.T.; Gorroochurn, P.; Redler, L.H.; Schiu, B.; Popkin, C.A. Epidemiology of Foot and Ankle Injuries in National Collegiate Athletic Association Men's and Women's Ice Hockey. Orthop. J. Sports Med. 2019, 7, 2325967119865908. [CrossRef]

14. Comstock, R.; Currie, D.; Pierpoint, L. Convenience Sample Summary Report: National High School Sports-Related Injury Surveillance Study: 2008-2018 School Years. Available online: http://www.ucdenver.edu/academics/colleges/PublicHealth/research/ResearchProjects/piper/projects/ RIO/Pages/Study-Reports.aspx (accessed on 21 October 2019).

15. Emery, C.; Kang, J.; Shrier, I.; Goulet, C.; Hagel, B.; Benson, B.; Nettel-Aguirre, A.; McAllister, J.; Meeuwisse, W. Risk of injury associated with bodychecking experience among youth hockey players. CMAJ 2011, 183, 1249-1256. [CrossRef]

16. Torraco, R.J. Writing integrative literature reviews: Using the past and present to explore the future. Hum. Resour. Dev. Rev. 2016, 15, 404-428. [CrossRef]

17. Falagas, M.E.; Pitsouni, E.I.; Malietzis, G.A.; Pappas, G. Comparison of PubMed, Scopus, web of science, and Google scholar: Strengths and weaknesses. FASEB J. 2008, 22, 338-342. [CrossRef]

18. Lorentzon, R.; Wedren, H.; Pietilä, T.; Gustavsson, B. Injuries in international ice hockey: A prospective, comparative study of injury incidence and injury types in international and Swedish elite ice hockey. Am. J. Sports Med. 1988, 16, 389-391. [CrossRef]

19. Flik, K.; Lyman, S.; Marx, R.G. American collegiate men's ice hockey: An analysis of injuries. Am. J. Sports Med. 2005, 33, 183-189. [CrossRef]

20. Pelletier, R.L.; Montelpare, W.J.; Stark, R.M. Intercollegiate ice hockey injuries: A case for uniform definitions and reports. Am. J. Sports Med. 1993, 21, 78-81. [CrossRef]

21. Stuart, M.J.; Smith, A. Injuries in junior A ice hockey: A three-year prospective study. Am. J. Sports Med. 1995, 23, 458-461. [CrossRef] 
22. Tuominen, M.; Stuart, M.J.; Aubry, M.; Kannus, P.; Parkkari, J. Injuries in world junior ice hockey championships between 2006 and 2015. Br. J. Sports Med. 2017, 51, 36-43. [CrossRef]

23. McKnight, C.M.; Ferrara, M.S.; Czerwinska, J.M. Intercollegiate ice hockey injuries: A three-year analysis. J. Athl. Train. 1992, 27, 338-343.

24. Tegner, Y.; Lorentzon, R. Ice hockey injuries: Incidence, nature and causes. Br. J. Sports Med. 1991, 25, 87-89. [CrossRef]

25. Hayes, D. Hockey injuries: How, why, where, and when? Physician Sports Med. 1975, 3, 61-65. [CrossRef]

26. Sutherland, G.W. Fire on ice. Am. J. Sports Med. 1976, 4, 264-269. [CrossRef]

27. Hayes, D. An injury profile for hockey. Can. J. Appl. Sport Sci. 1978, 3, 61-64.

28. Rielly, M. The nature and causes of hockey injuries: A five year study. Athl. Train. 1982, 17, 88-90.

29. Meeuwisse, W.H.; Fowler, P.J. Frequency and predictability of sports injuries in intercollegiate athletes. Canad. J. Sport Sci. 1988, 13, 35-42.

30. Lorentzon, R.; Wedrèn, H.; Pietilä, T. Incidence, nature, and causes of ice hockey injuries: A three-year prospective study of a Swedish elite ice hockey team. Am. J. Sports Med. 1988, 16, 392-396. [CrossRef]

31. Pettersson, M.; Lorentzon, R. Ice hockey injuries: A 4-year prospective study of a Swedish elite ice hockey team. Br. J. Sports Med. 1993, 27, 251-254. [CrossRef]

32. Cunningham, C.; Cunningham, S. Injury surveillance at a national multi-sport event. Aust. J. Sci. Med. Sport 1996, 28, 50-56. [PubMed]

33. Mölsä, J.; Airaksinen, O.; Näsman, O.; Torstila, I. Ice hockey injuries in Finland: A prospective epidemiologic study. Am. J. Sports Med. 1997, 25, 495-499. [CrossRef] [PubMed]

34. Pinto, M.; Kuhn, J.E.; Greenfield, M.; Hawkins, R.J. Prospective analysis of ice hockey injuries at the junior A level over the course of one season. Clin. J. Sport Med. 1999, 9, 70-74. [CrossRef] [PubMed]

35. Mölsä, J.; Kujala, U.; Näsman, O.; Lehtipuu, T.-P.; Airaksinen, O. Injury profile in ice hockey from the 1970s through the 1990s in Finland. Am. J. Sports Med. 2000, 28, 322-327. [CrossRef]

36. Agel, J.; Dompier, T.P.; Dick, R.; Marshall, S.W. Descriptive epidemiology of collegiate men's ice hockey injuries: National Collegiate Athletic Association Injury Surveillance System, 1988-1989 through 2003-2004. J. Athl. Train. 2007, 42, 241-248.

37. Rishiraj, N.; Lloyd-Smith, R.; Lorenz, T.; Niven, B.; Michel, M. University men's ice hockey: Rates and risk of injuries over 6-years. J. Sports Med. Phys. Fitness 2009, 49, 159-166.

38. Kuzuhara, K.; Shimamoto, H.; Mase, Y. Ice hockey injuries in a Japanese elite team: A 3-year prospective study. J. Athl. Train. 2009, 44, 208-214. [CrossRef]

39. Agel, J.; Harvey, E.J. A 7-year review of men's and women's ice hockey injuries in the NCAA. Can. J. Surg. 2010, 53, 319-323.

40. Engebretsen, L.; Steffen, K.; Alonso, J.M.; Aubry, M.; Dvorak, J.; Junge, A.; Meeuwisse, W.; Mountjoy, M.; Renstrom, P.; Wilkinson, M. Sports injuries and illnesses during the Winter Olympic Games 2010. Br. J. Sports Med. 2010, 44, 772-780. [CrossRef]

41. Kerr, Z.Y.; Dompier, T.P.; Snook, E.M.; Marshall, S.W.; Klossner, D.; Hainline, B.; Corlette, J. National Collegiate Athletic Association Injury Surveillance System: Review of methods for 2004-2005 through 2013-2014 data collection. J. Athl. Train. 2014, 49, 552-560. [CrossRef]

42. Tuominen, M.; Stuart, M.J.; Aubry, M.; Kannus, P.; Parkkari, J. Injuries in men's international ice hockey: A 7-year study of the International Ice Hockey Federation Adult World Championship Tournaments and Olympic Winter Games. Br. J. Sports Med. 2015, 49, 30-36. [CrossRef]

43. Kerr, Z.Y.; Marshall, S.W.; Dompier, T.P.; Corlette, J.; Klossner, D.A.; Gilchrist, J. College Sports-Related Injuries-United States, 2009-10 Through 2013-14 Academic Years. MMWR Morb. Mortal. Wkly. Rep. 2015, 64, 1330-1336. [CrossRef]

44. Lynall, R.C.; Mihalik, J.P.; Pierpoint, L.A.; Currie, D.W.; Knowles, S.B.; Wasserman, E.B.; Dompier, T.P.; Comstock, R.D.; Marshall, S.W.; Kerr, Z.Y. The First Decade of Web-Based Sports Injury Surveillance: Descriptive Epidemiology of Injuries in US High School Boys' Ice Hockey (2008-2009 Through 2013-2014) and National Collegiate Athletic Association Men's and Women's Ice Hockey (2004-2005 Through 2013-2014). J. Athl. Train. 2018, 53, 1129-1142.

45. Clarsen, B.; Bahr, R. Matching the choice of injury/illness definition to study setting, purpose and design: One size does not fit all! Br. J. Sports Med. 2014, 48, 510-512. [CrossRef] 
46. Timpka, T.; Alonso, J.-M.; Jacobsson, J.; Junge, A.; Branco, P.; Clarsen, B.; Kowalski, J.; Mountjoy, M.; Nilsson, S.; Pluim, B. Injury and illness definitions and data collection procedures for use in epidemiological studies in Athletics (track and field): Consensus statement. Br. J. Sports Med. 2014, 48, 483-490. [CrossRef]

47. MacDonald, K.J.; Palacios-Derflingher, L.M.; Emery, C.A.; Meeuwisse, W.H. The Effect of Injury Definition and Surveillance Methodology on Measures of Injury Occurrence and Burden in Elite Volleyball. Int. J. Sports Med. 2018, 39, 860-866. [CrossRef]

48. Orchard, J.; Hoskins, W. For debate: Consensus injury definitions in team sports should focus on missed playing time. Clin. J. Sport Med. 2007, 17, 192-196. [CrossRef]

49. Dick, R.; Agel, J.; Marshall, S.W. National Collegiate Athletic Association injury surveillance system commentaries: Introduction and methods. J. Athl. Train. 2007, 42, 173-182.

50. Longstaffe, R.; Leiter, J.; MacDonald, P. Anterior Cruciate Ligament Injuries in the National Hockey League: Epidemiology and Performance Impact. Clin. J. Sport Med. 2018, in press. [CrossRef]

51. Orchard, J.; Newman, D.; Stretch, R.; Frost, W.; Mansingh, A.; Lelpus, A. Methods for injury surveillance in international cricket. South. Afr. J. Sports Med. 2005, 17, 18-28.

52. Orchard, J.W.; Seward, H.; Orchard, J.J. Results of 2 decades of injury surveillance and public release of data in the Australian Football League. Am. J. Sports Med. 2013, 41, 734-741. [CrossRef] [PubMed]

53. West, S.W.; Williams, S.; Kemp, S.; Cross, M.J.; Stokes, K.A. Athlete Monitoring in Rugby Union: Is Heterogeneity in Data Capture Holding Us Back? Sports 2019, 7, 98. [CrossRef] [PubMed]

(C) 2019 by the authors. Licensee MDPI, Basel, Switzerland. This article is an open access article distributed under the terms and conditions of the Creative Commons Attribution (CC BY) license (http://creativecommons.org/licenses/by/4.0/). 